\title{
Migraine treatment and healthcare costs: retrospective analysis of the China Health Insurance Research Association (CHIRA) database
}

Shengyuan $\mathrm{Yu}^{1}$, Yanlei Zhang ${ }^{2^{*}}$, Yuan $\mathrm{YaO}^{2}$ and Haijun $\mathrm{CaO}^{2}$

\begin{abstract}
Background: Adult migraine remains underdiagnosed and undertreated, despite significant negative effects on physical and emotional functioning. Information on prescribing patterns and treatment costs of migraine in China is limited.

Methods: This retrospective analysis of the China Health Insurance Research Association (CHIRA) medical insurance claims database in 2016 to 2017 evaluated treatment patterns, direct medical costs, and healthcare resource utilization among adults with migraine in mainland China.

Results: Of 108,375 patients with headache-related outpatient visits, 10,652 were adults with migraine (mean age 51.4 years, $55.4 \%$ female). Common comorbidities were major depressive disorder (4.1\%), insomnia (3.8\%), and anxiety (2.3\%). Migraine patients were prescribed acute medication (26.4\%), preventive medication (15.0\%), and Chinese patent and herbal medicines (24.5\% and $11.7 \%$, respectively). Of patients prescribed acute medication, $68.8 \%$ received non-aspirin non-steroidal anti-inflammatory drugs (NSAIDs), 7.1\% received opioids, while only 3.3\% received triptans. Mean annual outpatient costs per patient were 46.5 United States dollars (USD), with mean (standard deviation) 1.8 (2.0) outpatient visits per year. Medication costs for traditional Chinese medicine (22.4 USD per patient) were higher than for Western medicine (13.5 USD).
\end{abstract}

Conclusion: Among migraine patients in China, NSAIDs were commonly prescribed as acute medication, while utilization of migraine-specific triptans and preventive medications was low.

Keywords: Migraine disorder, Adult, Drug prescriptions, Acute medication, Preventive medication, Retrospective studies

\section{Background}

Migraine is a primary headache disorder characterized by disabling attacks of moderate-to-severe pain, accompanied by nausea, photophobia, or phonophobia, which impair ability to function and adversely impact quality of

\footnotetext{
* Correspondence: zhang_yan_lei@lilly.com

${ }^{2}$ Lilly Suzhou Pharmaceutical Co., Ltd. Shanghai Branch, 19F, Centre T1, HKRI Taikoo, No. 288, Shimen No.1 Road, Shanghai 200021, China

Full list of author information is available at the end of the article
}

life [1-3]. Migraine has an estimated global prevalence of $14.4 \%$ and is the second highest cause of years lived with disability based on the Global Burden of Disease Study [3].

In China, population-based estimates of the 1-year prevalence of migraine range from $7.9 \%$ to $14.3 \%$ in adults [4-6]. Health-related quality-of-life studies in China, including Taiwan, have demonstrated substantial negative effects of migraine on physical and emotional

C C The Author(s). 2020 Open Access This article is licensed under a Creative Commons Attribution 4.0 International License, which permits use, sharing, adaptation, distribution and reproduction in any medium or format, as long as you give appropriate credit to the original author(s) and the source, provide a link to the Creative Commons licence, and indicate if changes were made. The images or other third party material in this article are included in the article's Creative Commons licence, unless indicated otherwise in a credit line to the material. If material is not included in the article's Creative Commons licence and your intended use is not permitted by statutory regulation or exceeds the permitted use, you will need to obtain permission directly from the copyright holder. To view a copy of this licence, visit http://creativecommons.org/licenses/by/4.0/ The Creative Commons Public Domain Dedication waiver (http://creativecommons.org/publicdomain/zero/1.0/) applies to the data made available in this article, unless otherwise stated in a credit line to the data. 
functioning, impacting work, household, and leisure activities [5, 7-9]. In a population of 1.4 billion, migraine presents a significant clinical burden in China.

Migraine treatment guidelines for China are consistent with those for Europe and the United States (US) in supporting a stratified approach to acute (abortive) treatment, whereby medication choice is based on attack severity and symptoms, the efficacy and side effect profile of the drug, and the patient's previous response to acute treatment [10-12]. Chinese guidelines recommend non-steroidal anti-inflammatory drugs (NSAIDs), acetaminophen, compound analgesics containing caffeine (cautioning that caffeine could increase the risk of drug addiction and medication overuse headache), and triptans; opioids, barbiturates, and ergotamine derivatives are not recommended for regular use [10].

Preventive (prophylaxis) treatment can be offered in the case of recurring migraine that is interfering with daily routine despite acute treatment; failure of, contraindication to, or side effects from acute medications; risk of acute medication overuse; circumstances such as hemiplegic migraine; high or increasing attack frequency; and patient preference [13]. Metoprolol, propranolol, flunarizine, valproic acid, and topiramate are the recommended preventives based on Level A evidence in Chinese and European guidelines [10, 11]. An estimated $34 \%$ to $39 \%$ of patients with migraine in Europe and the US are eligible for preventive migraine treatment $[14,15]$. However, in the European Eurolight study, less than $14 \%$ of eligible patients actually received preventive medication, and in the American Migraine Prevalence and Prevention (AMPP) study, 32\% of neverusers of preventive treatment would benefit from their use, suggesting undertreatment and inadequate disease management $[14,15]$.

Information on the prescribing patterns and treatment costs of migraine in mainland China is limited. Although there is substantial evidence for the economic burden of migraine in the US and Europe, this information cannot be generalized to China because of the differences in diagnosis and treatment practices, availability of prescription medication, and use of traditional Chinese medicine alongside Western medicine. A populationbased survey in China in 2009 estimated the total annual direct costs of migraine diagnosis and treatment to be 58.0 billion Chinese yuan renminbi (CNY) (8.4 billion US dollars [USD]) [5]. However, this survey relied on patient-reported information for migraine diagnosis and healthcare resource use. Hence, there is a need for China-specific data reflecting local diagnostic and treatment practices and the use of complementary medicine.

This retrospective analysis of the China Health Insurance Research Association (CHIRA) nationwide medical insurance claims data aimed to understand the treatment patterns, direct medical costs, and healthcare resource utilization among adult patients with migraine in mainland China.

\section{Methods \\ Dataset}

The CHIRA database is a medical insurance management information system initiated in 2007 that contains nationwide consecutive inpatient and outpatient visit claims data of urban basic medical insurance in China. With approximately $95 \%$ of the population in mainland China covered by public medical insurance, the CHIRA database is a valuable resource of real-world evidence for medical costs in China [16]. CHIRA data are collected annually from the local insurance centers of a random de-identified sample of selected areas of China including at least $2 \%$ of municipalities, at least $2 \%$ of provincial capital cities, and at least $5 \%$ of prefecturelevel cities. The database contains patient basic information, medical institution information, diagnosis results, healthcare service utilization, medication prescription, and healthcare expenditure details.

CHIRA granted permission to access the anonymized database for the purposes of this study. Only anonymized information was accessible from the CHIRA database and therefore institutional ethics approval and informed consent were not required.

\section{Patient selection}

All outpatient visit and healthcare use information for the diagnosis and treatment of headache in 2016 and 2017 was extracted from the CHIRA database. Adult patients ( $\geq 18$ years) were selected who had a primary diagnosis of migraine, identified by International Statistical Classification of Diseases and Related Health Problems revision 10 (ICD-10) code (ICD-10 G43.0, G43.1, G43.2, G43.3, G43.8, G43.9) and supplemented by searching physician Chinese character descriptions against translations from the World Health Organization Chinese version of ICD-10. Patients were excluded if they had cluster headache (ICD-10 G44.0), malignancy, schizophrenia, or stroke, or were receiving hemodialysis or peritoneal dialysis.

\section{Outcome measures}

Patient information identified from the CHIRA database included age, gender, comorbidities (predefined as anxiety, major depressive disorder, epilepsy, fremitus, and insomnia), and medical insurance type (Urban Employee Basic Medical Insurance or Urban Residents Basic Medical Insurance).

Medications prescribed in the 2016-2017 period for the treatment of migraine or for pain relief were identified and categorized according to class of acute 
medication (including aspirin, paracetamol, nonaspirin NSAIDs, opioids, triptans, ergot alkaloids, barbiturates, antiemetics, glucocorticoids, and mannitol) and preventive medication (calcium antagonists, $\beta 1$ receptor blockers, antiepileptics, antidepressants, and type A botulinum toxin). Medications within each acute and preventive medication category are listed in Additional Table 1. Prescriptions for Chinese patent and herbal medicines were identified using Chinese character search terms. Chinese patent medicines are manufactured products with a standardized composition of herbal extracts and other ingredients, generally available as pills, capsules, or liquids. Chinese patent medicines used in the treatment of migraine generally contain extracts such as Ligusticum wallichii, Angelica dahurica root, and Gastrodia elata, with the aim of promoting circulation and relieving pain. Chinese herbal medicines are herbal decoctions, often condensed into granules or powder.

Healthcare resource utilization included the hospital level and department of the patient's first migrainerelated visit in the 2016-2017 period. Diagnostic methods were identified using Chinese character keyword searches for cranial computed tomography (CT), cranial magnetic resonance imaging (MRI), transcranial Doppler ultrasonography, and electroencephalography (EEG).
Annual visit frequency and direct medical costs were calculated in the subgroup of patients with a migraine-related outpatient visit in January of 2016 or 2017 and with at least 11 months of follow-up. Medication costs were calculated for prescribed Western medicine and for Chinese patent or herbal medicine. Diagnostic costs included claims for cranial CT, cranial MRI, transcranial Doppler ultrasonography, and EEG. Non-medication costs included physical therapy and oxygen treatment.

\section{Statistical analysis}

Study variables were summarized using descriptive statistics. Analyses were performed using STATA/SE 14.0 (StataCorp) software (College Station, TX: StataCorp LP).

\section{Results}

\section{Patient population and characteristics}

Data from over 16 million patients were retrieved from the CHIRA outpatient database in the calendar years of 2016 and 2017. A total of 108,375 patients had headache-related outpatient visits, of whom 10,652 adult patients with migraine met the eligibility criteria and were included in the analysis (Fig. 1).

Mean age was 51.4 years (standard deviation [SD] 15.8 years) and $55.4 \%$ of patients were female (Table 1 ).

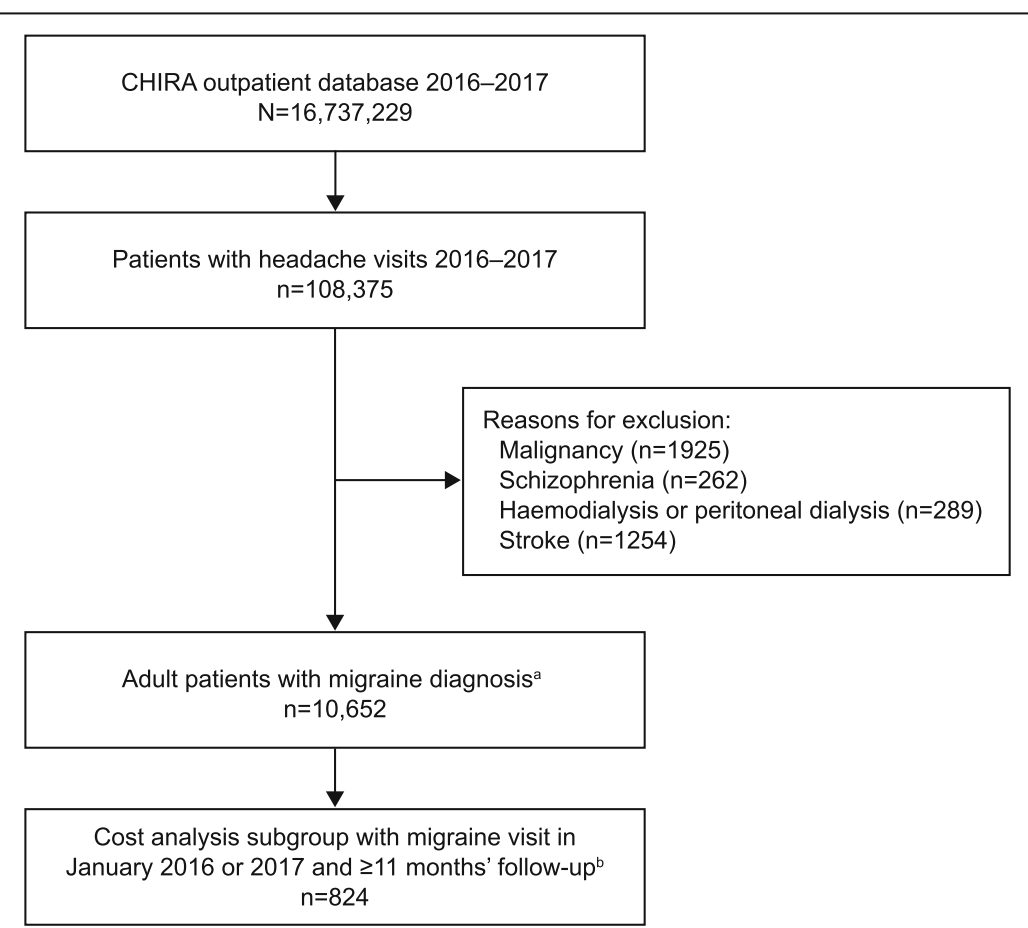

Fig. 1 Patient selection. ${ }^{a}$ Migraine diagnosis by ICD-10 code (ICD-10 G43.0, G43.1, G43.2, G43.3, G43.8, G43.9) supplemented by physician Chinese characters descriptions. ${ }^{b}$ Patients with a migraine-related outpatient visit in January 2016 or January 2017 and with $\geq 11$ months of follow-up were included in the outpatient cost analysis. CHIRA: China Health Insurance Research Association; ICD: International Statistical Classification of Diseases and Related Health Problems 
Table 1 Patient characteristics

\begin{tabular}{|c|c|}
\hline & $\begin{array}{l}\text { Adults with migraine } \\
(N=10,652)\end{array}$ \\
\hline Age, years, mean (SD) & $51.4(15.8)$ \\
\hline \multicolumn{2}{|l|}{ Age category, years, n (\%) } \\
\hline $18-29$ & $958(9.0)$ \\
\hline $30-39$ & $1736(16.3)$ \\
\hline $40-49$ & $2222(20.9)$ \\
\hline $50-59$ & $2243(21.1)$ \\
\hline$\geq 60$ & $3493(32.8)$ \\
\hline Female, n (\%) & $5902(55.4)$ \\
\hline \multicolumn{2}{|l|}{ Insurance type, n (\%) } \\
\hline UEBMI & $7675(72.1)$ \\
\hline URBMI & $2977(27.9)$ \\
\hline \multicolumn{2}{|l|}{ Comorbidities ${ }^{a}, \mathrm{n}(\%)$} \\
\hline Major depressive disorder & $442(4.1)$ \\
\hline Insomnia & $402(3.8)$ \\
\hline Anxiety & $248(2.3)$ \\
\hline Epilepsy & $46(0.4)$ \\
\hline Fremitus & $17(0.2)$ \\
\hline Hospital level of first visit ${ }^{\mathrm{b}}, \mathrm{n}(\%)$ & $(n=9982)$ \\
\hline Primary & $6686(67.0)$ \\
\hline Secondary & $1384(13.9)$ \\
\hline Tertiary & $1912(19.2)$ \\
\hline Department of tertiary hospital first visit ${ }^{\mathrm{b}}, \mathrm{n}(\%)$ & $(n=979)$ \\
\hline Neurology & $402(41.1)$ \\
\hline Traditional Chinese medicine & $130(13.3)$ \\
\hline Internal medicine & $128(13.1)$ \\
\hline Emergency room & $68(6.9)$ \\
\hline Other & $251(25.6)$ \\
\hline
\end{tabular}

CHIRA China Health Insurance Research Association, SD standard deviation, UEBMI Urban Employee Basic Medical Insurance, URBMI Urban Residents Basic Medical Insurance

a Incidence of predefined comorbidities recorded in the period 2016-2017 in the CHIRA database

${ }^{\mathrm{b}}$ First migraine-related visit in the period 2016-2017 in the CHIRA database

Comorbidities included major depressive disorder (4.1\%), insomnia (3.8\%), and anxiety (2.3\%). Of patients for whom first migraine-related visit site was recorded, over two-thirds visited a primary-level hospital; $19.2 \%$ visited a tertiary-level hospital, most commonly to a neurology department.

\section{Prescribed migraine medication}

Acute medication was prescribed for 2813 (26.4\%) patients (Table 2). The majority (68.8\%) of patients receiving acute medication were prescribed non-aspirin NSAIDs, with ibuprofen being the most common (36.5\%). Other classes of acute medication were each
Table 2 Acute medication prescribed for migraine

\begin{tabular}{ll}
\hline & $\begin{array}{l}\text { Adults with migraine } \\
(N=10,652)\end{array}$ \\
\hline Patients prescribed $\geq 1$ acute medication, $\mathrm{n}(\%)$ & $2813(26.4)$ \\
Types of acute medication, $\mathrm{n}(\%)$ & $(n=2813)$ \\
Non-aspirin NSAID & $1934(68.8)$ \\
Aspirin & $225(8.0)$ \\
Weak opioids/opioids & $199(7.1)$ \\
Ergot alkaloids & $172(6.1)$ \\
Acetaminophen & $122(4.3)$ \\
Triptans & $92(3.3)$ \\
Antiemetics & $53(1.9)$ \\
Others & $199(7.1)$ \\
\hline
\end{tabular}

NSAID non-steroidal anti-inflammatory drug

prescribed to $\leq 8.0 \%$ of patients receiving acute medication, including opioids (7.1\%) and ergot alkaloids (6.1\%). Only $3.3 \%$ of patients receiving acute medication were prescribed a triptan.

Preventive medication was prescribed for 1602 (15.0\%) patients (Table 3). Of patients receiving preventive medication, the majority $(88.3 \%)$ were prescribed calcium antagonists, primarily flunarizine $(87.6 \%)$, and $8.4 \%$ were prescribed $\beta 1$-receptor antagonists. Other classes were each prescribed to less than $4 \%$ of the patients receiving preventive medication.

Chinese patent medicine was prescribed for 2612 (24.5\%) patients and Chinese herbal medicine was prescribed for 1248 (11.7\%) patients. The proportions of patients prescribed Chinese patent or herbal medicine were comparable with those prescribed Western acute (26.4\%) or preventive (15.0\%) medications. Traditional Chinese medicines were prescribed at $64.3 \%$ of all migraine-related outpatient visits.

\section{Healthcare resource utilization and costs}

Mean annual number of outpatient visits per patient was 1.8 (SD 2.0; median, 1.0). Healthcare resources for

Table 3 Preventive medication prescribed for migraine

\begin{tabular}{ll}
\hline & $\begin{array}{l}\text { Adults with migraine } \\
(N=10,652)\end{array}$ \\
\hline $\begin{array}{l}\text { Patients prescribed } \geq 1 \text { preventive } \\
\text { medication, } \mathrm{n}(\%)\end{array}$ & $1602(15.0)$ \\
Types of preventive medication, $\mathrm{n}(\%)$ & $(n=1602)$ \\
Calcium antagonists & $1414(88.3)$ \\
$\beta$ 1-receptor antagonists & $135(8.4)$ \\
Antiepileptics & $43(2.7)$ \\
Antidepressants & $25(1.6)$ \\
Type A botulinum toxin & $1(0.1)$ \\
Others & $54(3.4)$ \\
\hline
\end{tabular}


migraine diagnosis included cranial CT (4.2\% of visits), cranial MRI (1.8\%), transcranial Doppler ultrasonography (1.2\%), and EEG (0.1\%).

The cost analysis subgroup included 824 patients with a migraine-related outpatient visit in January of 2016 or 2017 and with at least 11 months of follow-up (Fig. 1). Mean annual outpatient costs per patient were 46.5 USD, with medication costs accounting for 36.0 USD and diagnostic and non-medication costs accounting for 10.5 USD (Table 4). Medication costs for traditional Chinese medicine (22.4 USD per patient) were higher than costs for Western medicine (13.5 USD).

\section{Discussion}

This is the first study to describe the treatment patterns and costs of migraine treatment in urban mainland China using a retrospective analysis of the large CHIRA medical insurance claims database. The treatment patterns for adult migraine patients in this study indicate relatively low levels of headache management in China. Only $26.4 \%$ of patients were prescribed acute medication, which contrasts with higher rates (73\%) from Japanese insurance claims data [17]; this may reflect cultural differences in prescribing patterns or greater reliance on over-the-counter rather than prescription acute medications in China. In the US, approximately half of patients used prescription acute medication, with or without over-the-counter acute medication [18], and much higher rates were reported among patients consulting specialist neurologists and physicians for their migraine [19].

Non-aspirin NSAIDs, primarily ibuprofen, were by far the most commonly prescribed acute medication in our study, which is in accordance with international treatment guidelines [10-12] and may also reflect their accessibility and relatively low cost. There is evidence to support ibuprofen as effective for severe migraine attacks; however, NSAIDs are non-migraine-specific, and

Table 4 Annual outpatient costs per patient

\begin{tabular}{lll}
\hline $\mathrm{N}=824^{\mathrm{a}}$ & Costs, USD $^{\mathrm{b}}$ & \\
\cline { 2 - 3 } & Mean (SD) & Median \\
\hline Total annual costs per patient & $46.5(80.8)$ & 20.6 \\
Medication costs & $36.0(73.7)$ & 15.9 \\
$\quad$ Western medicine & $13.5(23.4)$ & 6.5 \\
$\quad$ Traditional Chinese medicine & $22.4(43.3)$ & 7.6 \\
Diagnostic and non-medication costs $^{c}$ & $10.5(30.7)$ & 0.9 \\
\hline
\end{tabular}

USD United States dollars

a Patients with a migraine-related outpatient visit in January of 2016 or 2017 and with $\geq 11$ months of follow-up

${ }^{b}$ Exchange rate: 1 USD $=6.697$ Chinese yuan renminbi

${ }^{c}$ Migraine diagnostic costs included cranial computed tomography, cranial magnetic resonance imaging, transcranial Doppler ultrasonography, and electroencephalography; migraine non-medication costs included physical therapy and oxygen treatment their acute use can be associated with peptic ulcer or renal disease, which should be considered in treatment decisions [12].

Triptans are a class of recommended migraine-specific acute medication [10-12] and are also effective in about $60 \%$ of non-responders to NSAIDs [20]. Three types of triptan are commercially available in China: sumatriptan, zolmitriptan, and rizatriptan. Although the Level A evidence supporting triptan use is recognized in Chinese as well as international treatment guidelines [10-12], the rate of triptan prescribing in this study was low; of patients receiving acute medication, only $3.3 \%$ were prescribed triptans. Surveys of migraine patients attending hospital neurology departments in mainland China have also found triptan use to be rare [21, 22]. In contrast, triptans are among the most commonly prescribed acute medications for migraine in Europe, the US, and Japan $[17,19,23,24]$. The lower rate of triptan use in China may be explained by barriers to access owing to limited triptan availability in most hospitals and pharmacies. Limited over-the-counter purchase of triptans has also been reported in China, with their relatively high price making triptans unaffordable to many patients with migraine [22].

In our study, opioids were prescribed for $7.1 \%$ of patients receiving acute medication. In contrast, more patients are prescribed opioids for migraine in the US, from $16 \%$ in the AMPP study [25] to $36 \%$ in the Chronic Migraine Epidemiology and Outcomes study [26]. The consumption of opioids in China is far lower than the global average, with a mean defined daily opioid dose in 2011 to 2013 of 96 units per million people per day in China compared with 3027 units globally [27]. Opioids are subject to government regulation in China [28] and are not recommended for the acute treatment of $\mathrm{mi}$ graine in Chinese, US, or European guidelines [10-12] due to the potential for medication overuse and transformation from episodic to chronic migraine [29, 30]. Although we cannot determine whether the $7.1 \%$ of patients who were prescribed opioids in our study were non-responders to other medications, this rate of opioid prescription demonstrates an unmet need for additional migraine-specific acute treatments that lack the safety concerns of opioids.

Preventive medication was prescribed for only $15 \%$ of patients in our study, consistent with insurance claims data for Japan, which showed that $15 \%$ of patients received preventive treatment. However, these rates are well below the estimated 34\% to 39\% of patients who would benefit from migraine preventive treatment reported for Europe and the US [14, 15]. The limited available evidence from other studies in China also suggests that preventive medications are underutilized, with $\leq 5 \%$ of patients receiving preventive medication [21, 31]. In 
our study, the calcium-channel blocker flunarizine was the most commonly prescribed preventive, used by $87.6 \%$ of patients receiving preventives. The preferential use of flunarizine over other migraine preventives in China may reflect its relatively mild adverse effects, which can include fatigue, weight gain, and mood changes [32].

Approximately one quarter of patients in our study were prescribed Chinese patent medicine and over $11 \%$ were prescribed Chinese herbal medicine. Moreover, the mean annual direct costs for traditional Chinese medicine (patent or herbal) were almost twice the estimated direct cost of prescribed Western medicine. Although a meta-analysis has suggested efficacy for Chinese patent medicines in the treatment of migraine, many available studies are of poor methodological quality and the potential mechanisms of action are unclear [33]. This is consistent with the finding by Luo et al. of lower rates of satisfaction with Chinese patent medicine compared with Western medicine in migraine treatment [22]. Given the high direct costs of traditional Chinese medicine shown in our study and potentially low rates of satisfaction, there is a need for greater understanding of their efficacy in migraine treatment and the factors driving the prescription of traditional Chinese medicine in preference to Western medicine.

The mean total annual outpatient costs for medication, diagnostic tests, and non-medication costs for physical therapy or oxygen treatment were estimated to be 46.5 USD per patient. This is lower than the annual direct costs previously reported by $\mathrm{Yu}$ et al. for migraine diagnosis and treatment in China, which were estimated to be 105 USD per patient [5]. This difference may reflect study design differences, with $\mathrm{Yu}$ et al. including all outof-pocket expenses for diagnosis and treatment, in contrast with our study, which included medical insurance claims and excluded over-the-counter purchases from pharmacies. A retrospective case-control analysis of the Taiwanese nationwide National Health Insurance Research Database estimated annual total drug costs from outpatient visits to be significantly higher for patients with refractory migraine compared with that for nonmigraine control subjects, but the estimated costs were higher than reported in our study owing to the inclusion of costs associated with comorbid conditions [34].

The strengths of the current study include the wide urban population coverage of the CHIRA database. Approximately $95 \%$ of China's population is covered by public health insurance [16], and China's urban population represents approximately $59 \%$ of the total population [35]. CHIRA data also represent a range of localities, including municipalities, provincial capital cities, and prefecture-level cities, and include primarythrough to tertiary-level hospitals. However, the findings may not be representative of patients in rural areas or those without urban employee or resident basic medical insurance. Patients who did not seek treatment from a healthcare provider and used only over-the-counter medications would also be excluded from the study. In addition, the database does not include medications purchased outside of hospital pharmacies by the enrolled patients and is therefore likely to underestimate medication use. Only patients with a primary diagnosis of migraine were included in the analysis; however, some medications (eg, calcium antagonists) may have been prescribed for other indications, resulting in an overestimation of certain prescriptions for migraine. Lastly, although it was not possible to determine the incidence of chronic and episodic migraine in this population, the cost analysis was based on actual prescription data and, therefore, would reflect the potentially more frequent use of medication in patients with chronic migraine.

\section{Conclusions}

Our findings from the analysis of this large nationwide database are a representative reflection of migraine treatment and costs in urban mainland China. Prescribing rates for acute medication in China were low compared with the US and Japan. NSAIDs were the most commonly prescribed acute medication, while there was low utilization of migraine-specific triptans and preventive medications. Traditional Chinese medicine was commonly prescribed in clinical practice, and with the costs of traditional Chinese medicine comprising the largest proportion of total drug costs. The findings indicate an economic burden associated with the diagnosis and treatment of migraine in China, and possible unmet needs for effective migraine-specific acute treatment as well as preventive medication.

\section{Supplementary information}

Supplementary information accompanies this paper at https://doi.org/10. 1186/s10194-020-01117-2.

Additional file 1: Table 1. Predefined search terms for prescription medication.

\section{Abbreviations \\ AMPP: American Migraine Prevalence and Prevention; CHIRA: China Health Insurance Research Association; CNY: Chinese yuan renminbi; CT: Computed tomography; EG: Electroencephalography; ICD-10: International Statistical Classification of Diseases and Related Health Problems revision 10; MRI: Magnetic resonance imaging; NSAID: Non-steroidal anti-inflammatory drug; SD: Standard deviation; UEBMI: Urban Employee Basic Medical Insurance; URBMI: Urban Residents Basic Medical Insurance; US: United States; USD: United States dollars}

\section{Acknowledgements}

Medical writing assistance was provided by Serina Stretton, PhD, CMPP and Linda Donnini, PhD, CMPP of ProScribe - Envision Pharma Group, and was funded by Eli Lilly and Company. ProScribe's services complied with international guidelines for Good Publication Practice (GPP3). 


\section{Authors' contributions}

All authors participated in the interpretation of study results, and in the drafting, critical revision, and approval of the final version of the manuscript. SY and YZ were involved in the study design.

\section{Funding}

This work was supported by Eli Lilly and Company, who was involved in the study design and preparation of the manuscript.

\section{Availability of data and materials}

The dataset for the current study is sampled from a database of nationwide claims data of urban basic medical insurance, and is not publicly available. The data analysis report is however available from the authors upon reasonable request.

\section{Ethics approval and consent to participate}

Only anonymized information was accessible from the CHIRA database and therefore institutional ethics approval and informed consent were not required.

\section{Consent for publication}

Not applicable.

\section{Competing interests}

YZ, YY, and HC are employees of Lilly Suzhou Pharmaceutical Co., Ltd., an affiliate of Eli Lilly and Company. SY has no conflicts of interest to declare.

\section{Author details}

'Department of Neurology, Chinese PLA General Hospital, Beijing, China. ${ }^{2}$ Lilly Suzhou Pharmaceutical Co., Ltd. Shanghai Branch, 19F, Centre T1, HKRI Taikoo, No. 288, Shimen No.1 Road, Shanghai 200021, China.

\section{Received: 5 March 2020 Accepted: 28 April 2020}

Published online: 13 May 2020

\section{References}

1. Headache Classification Committee of the International Headache Society (IHS) (2018) The International Classification of Headache Disorders, 3rd edition. Cephalalgia 38:1-211

2. Blumenfeld AM, Varon SF, Wilcox TK, Buse DC, Kawata AK, Manack A et al (2011) Disability, HRQoL and resource use among chronic and episodic migraineurs: results from the international burden of migraine study (IBMS). Cephalalgia 31:301-305

3. GBD 2016 Headache Collaborators (2018) Global, regional, and national burden of migraine and tension-type headache, 1990-2016: a systematic analysis for the Global Burden of Disease Study 2016. Lancet Neurol 17:954-976

4. Luo N, Qi W, Tong W, Tan F, Zhang Q, He J et al (2014) Prevalence and burden of headache disorders in two neighboring provinces of China. J Clin Neurosci 21:1750-1754

5. Yu S, Liu R, Zhao G, Yang X, Qiao X, Feng J et al (2012) The prevalence and burden of primary headaches in China: a population-based door-to-door survey. Headache 52:582-591

6. Gu X, Xie Y (2018) Migraine attacks among medical students in Soochow University, Southeast China: a cross-sectional study. J Pain Res 11:771-781

7. Wang X, Xing Y, Sun J, Zhou H, Yu H, Zhao Y et al (2016) Prevalence, associated factors, and impact on quality of life of migraine in a community in Northeast China. J Oral Facial Pain Headache 30:139-149

8. Wang SJ, Fuh JL, Lu SR, Juang KD (2001) Quality of life differs among headache diagnoses: analysis of SF-36 survey in 901 headache patients. Pain. 89:285-292

9. Hung PH, Fuh JL, Wang SJ (2006) Validity, reliability and application of the Taiwan version of the migraine disability assessment questionnaire. J Formos Med Assoc 105:563-568

10. Chinese Medical Association Group (2016) Guide to the prevention and treatment of migraine in China [Chinese]. Chin J Pain Med 22:721-727

11. Evers S, Afra J, Frese A, Goadsby PJ, Linde M, May A et al (2009) EFNS guideline on the drug treatment of migraine--revised report of an EFNS task force. Eur J Neurol 16:968-981

12. Marmura MJ, Silberstein SD, Schwedt TJ (2015) The acute treatment of migraine in adults: the American headache society evidence assessment of migraine pharmacotherapies. Headache 55:3-20
13. Parikh SK, Silberstein SD (2019) Preventive treatment for episodic migraine. Neurol Clin 37:753-770

14. Lipton RB, Bigal ME, Diamond M, Freitag F, Reed ML, Stewart WF (2007) Migraine prevalence, disease burden, and the need for preventive therapy. Neurology 68:343-349

15. Katsarava Z, Mania M, Lampl C, Herberhold J, Steiner TJ (2018) Poor medical care for people with migraine in Europe - evidence from the Eurolight study. J Headache Pain 19:10

16. Yang Y, Zhang J, Du F, Montgomery W, Li H, Flynn JA (2014) Real world evidence in mainland China: experience with the use of health care claims data. Value Health 17:A728

17. Meyers JL, Davis KL, Lenz RA, Sakai F, Xue F (2019) Treatment patterns and characteristics of patients with migraine in Japan: a retrospective analysis of health insurance claims data. Cephalalgia 39:1518-1534

18. Diamond S, Bigal ME, Silberstein S, Loder E, Reed M, Lipton RB (2007) Patterns of diagnosis and acute and preventive treatment for migraine in the United States: results from the American Migraine Prevalence and Prevention study. Headache 47:355-363

19. Ford JH, Jackson J, Milligan G, Cotton S, Ahl J, Aurora SK (2017) A real-world analysis of migraine: a cross-sectional study of disease burden and treatment patterns. Headache 57:1532-1544

20. Diamond ML, Hettiarachchi J, Hilliard B, Sands G, Nett R (2004) Effectiveness of eletriptan in acute migraine: primary care for Excedrin nonresponders. Headache 44:209-216

21. Li X, Zhou J, Tan G, Wang Y, Ran L, Chen L (2012) Diagnosis and treatment status of migraine: a clinic-based study in China. J Neurol Sci 315:89-92

22. Luo N, Qi W, Zhuang C, Di W, Lu Y, Huang Z et al (2014) A satisfaction survey of current medicines used for migraine therapy in China: is Chinese patent medicine effective compared with Western medicine for the acute treatment of migraine? Pain Med 15:320-328

23. Bigal ME, Borucho S, Serrano D, Lipton RB (2009) The acute treatment of episodic and chronic migraine in the USA. Cephalalgia 29:891-897

24. Vo P, Paris N, Bilitou A, Valena T, Fang J, Naujoks C et al (2018) Burden of migraine in Europe using self-reported digital diary data from the migraine buddy@ application. Neurol Ther 7:321-332

25. Buse DC, Pearlman SH, Reed ML, Serrano D, Ng-Mak DS, Lipton RB (2012) Opioid use and dependence among persons with migraine: results of the AMPP study. Headache 52:18-36

26. Schwedt TJ, Lipton RB, Friedman BW, Fanning KM, Reed MI (2019) Demographics, headache characteristics, and other factors associated with opioid use in people with migraine: results from the chronic migraine epidemiology and outcomes study. Headache 59:6-7

27. Fang W, Liu T, Gu Z, Li Q, Luo C (2019) Consumption trend and prescription pattern of opioid analgesics in China from 2006 to 2015. Eur J Hosp Pharm 26:140-145

28. Gu W (2015) Update on administration of anesthetics and psychoactive drugs for pain management in China. Acta Anaesthesiol Taiwanica 53:55-57

29. Trang T, Al-Hasani R, Salvemini D, Salter MW, Gutstein H, Cahill CM (2015) Pain and poppies: the good, the bad, and the ugly of opioid analgesics. J Neurosci 35:13879-13888

30. Bigal ME, Serrano D, Buse D, Scher A, Stewart WF, Lipton RB (2008) Acute migraine medications and evolution from episodic to chronic migraine: a longitudinal population-based study. Headache 48:1157-1168

31. Lu SR, Fuh JL, Chen WT, Juang KD, Wang SJ (2001) Chronic daily headache in Taipei, Taiwan: prevalence, follow-up and outcome predictors. Cephalalgia 21:980-986

32. Karsan N, Palethorpe D, Rattanawong W, Marin JC, Bhola R, Goadsby PJ (2018) Flunarizine in migraine-related headache prevention: results from 200 patients treated in the UK. Eur J Neurol 25:811-817

33. Xiao Y, Yuan L, Liu Y, Sun X, Cheng J, Wang T et al (2015) Traditional Chinese patent medicine for prophylactic treatment of migraine: a meta-analysis of randomized, double-blind, placebo-controlled trials. Eur J Neurol 22:361-368

34. Tang CH, Chen YC, Ng K, Wang SJ (2013) A retrospective matched casecontrol study on medical costs of refractory migraine in Taiwan. Headache 53:526-539

35. World Bank. World development indicators: urban population (\% of total population) in China. Available at: http://data.worldbank.org/indicator/SP. URB.TOTL.IN.ZS. Accessed 12 Aug 2019

\section{Publisher's Note}

Springer Nature remains neutral with regard to jurisdictional claims in published maps and institutional affiliations. 